\title{
Effects of a weight loss plus exercise program on physical function in overweight, older women: a randomized controlled trial
}

This article was published in the following Dove Press journal:

Clinical Interventions in Aging

I4 June 201 I

Number of times this article has been viewed

\author{
Stephen D Anton ${ }^{1,2}$ \\ Todd M Manini' \\ Vanessa A Milsom² \\ Pamela Dubyak ${ }^{2}$ \\ Matteo Cesari ${ }^{3}$ \\ Jing Cheng ${ }^{4}$ \\ Michael J Daniels ${ }^{5}$ \\ Michael Marsiske ${ }^{2}$ \\ Marco Pahor ${ }^{1}$ \\ Christiaan Leeuwenburgh' \\ Michael G Perri² \\ 'Department of Aging and Geriatric \\ Research, University of Florida, \\ Gainesville, FL, USA; ${ }^{2}$ Department \\ of Clinical and Health Psychology, \\ University of Florida, Gainesville, \\ FL, USA ${ }^{3}$ Area di Geriatria, Università \\ Campus Bio-Medico, Rome, Italy; \\ ${ }^{4}$ Division of Oral Epidemiology and \\ Dental Public Health, San Francisco, \\ CA, USA; ${ }^{5}$ Department of Statistics, \\ University of Florida, Gainesville, \\ FL, USA
}

Background: Obesity and a sedentary lifestyle are associated with physical impairments and biologic changes in older adults. Weight loss combined with exercise may reduce inflammation and improve physical functioning in overweight, sedentary, older adults. This study tested whether a weight loss program combined with moderate exercise could improve physical function in obese, older adult women.

Methods: Participants $(\mathrm{N}=34)$ were generally healthy, obese, older adult women (age range 55-79 years) with mild to moderate physical impairments (ie, functional limitations). Participants were randomly assigned to one of two groups for 24 weeks: (i) weight loss plus exercise (WL+E; $\mathrm{n}=17$; mean age $=63.7$ years [4.5]) or (ii) educational control $(\mathrm{n}=17$; mean age $=63.7$ [6.7]). In the WL+E group, participants attended a group-based weight management session plus three supervised exercise sessions within their community each week. During exercise sessions, participants engaged in brisk walking and lower-body resistance training of moderate intensity. Participants in the educational control group attended monthly health education lectures on topics relevant to older adults. Outcomes were: (i) body weight, (ii) walking speed (assessed by 400-meter walk test), (iii) the Short Physical Performance Battery (SPPB), and (iv) knee extension isokinetic strength.

Results: Participants randomized to the WL+E group lost significantly more weight than participants in the educational control group (5.95 [0.992] vs 0.23 [0.99] kg; $P<0.01)$. Additionally, the walking speed of participants in the WL+E group significantly increased compared with that of the control group (reduction in time on the 400-meter walk test $=44$ seconds; $P<0.05$ ). Scores on the SPPB improved in both the intervention and educational control groups from pre- to post-test $(P<0.05)$, with significant differences between groups $(P=0.02)$. Knee extension strength was maintained in both groups.

Conclusion: Our findings suggest that a lifestyle-based weight loss program consisting of moderate caloric restriction plus moderate exercise can produce significant weight loss and improve physical function while maintaining muscle strength in obese, older adult women with mild to moderate physical impairments.

Keywords: obesity, weight loss, physical function, oxidative stress, inflammation, walking speed

\section{Introduction}

In the past decade, the prevalence of obesity in older adults has doubled. ${ }^{1}$ Recent estimates indicate that an alarming $35 \%$ of adults aged 60 years and over are obese 2 and therefore are at increased risk for a number of health conditions, including cardiovascular disease, diabetes, hypertension, hyperlipidemia, asthma, osteoarthritis, and breast, prostate, and pancreatic cancer. ${ }^{3}$ Among older adults, African American women have the highest prevalence of obesity, with over half classified
Correspondence: Stephen D Anton 210 E. Mowry Road, Gainesville, FL, USA

$\mathrm{Tel}+13522737514$

Fax + I 3522735920

Email santon@aging.ufl.edu 
as obese, ${ }^{1}$ and also have disproportionately higher rates of weight-related comorbidities than do other older adult populations. ${ }^{4}$ Therefore, older African American women represent a particularly important, high-risk population. Obese, older adults are particularly susceptible to sarcopenia (the involuntary loss of skeletal muscle), and the combination of muscle loss and fat gain may act synergistically to increase risk for functional decline and physical disability. ${ }^{5-7}$

Despite these health risks, controversy exists in the treatment of obesity in older persons. ${ }^{89}$ Weight loss may improve mobility by reducing the load placed on the musculoskeletal system, but it could also adversely affect physical function by accelerating the rate of muscle loss that normally occurs with aging. ${ }^{5,6,10} \mathrm{~A}$ recent review of weight loss interventions in older adults concluded that weight loss interventions show a "modest but significant reduction in weight in older, obese people" but that "there is a paucity of outcome data that address issues directly relevant to older people - exercise capacity, physical function, and quality of life". ${ }^{11}$ Thus, the development of lifestyle-based weight loss interventions that can preserve muscle and improve physical function in older, obese adults represents a priority of paramount importance.

Although only a few studies have examined the effects of weight loss interventions that combine dietary restriction plus exercise in obese, older adults, the results of initial trials are encouraging. For example, the combination of diet-induced weight loss plus exercise was recently found to improve physical function and reverse frailty in obese, older adults ( $\geq 65$ years). ${ }^{12}$ Findings from another recent clinical trial suggest that the combination of aerobic plus resistance exercise may be more efficacious than aerobic exercise alone for improving physical function in overweight, older adults. ${ }^{13}$ Moreover, two other recent studies demonstrated that a diet plus exercise intervention can attenuate the loss of skeletal muscle that typically occurs from diet alone in obese, older adults. ${ }^{14,15}$ Thus, emerging literature suggests that weight loss interventions that incorporate exercise may have beneficial effects on physical function in obese, older adults.

To our knowledge, no study to date has examined the effects of a lifestyle-based weight loss plus exercise intervention involving both aerobic and resistance exercise in obese, older women with moderate physical limitations. We report here the effects of a 24-week lifestyle-based weight loss plus comprehensive exercise (WL+E) intervention on changes in body weight, physical function, and muscle strength in sedentary, obese, older African American and Caucasian women with mild to moderate physical limitations. We hypothesized that the WL+E intervention would produce significantly greater weight loss and larger improvements in physical function and strength compared with an educational control group.

\section{Methods}

\section{Participants}

Participants were sedentary, overweight, older, African American and Caucasian women with mild to moderate functional limitations. Eligibility requirements included age between 55 years and 79 years, body mass index $>28 \mathrm{~kg} / \mathrm{m}^{2}$, a sedentary lifestyle (defined as $<20 \mathrm{~min} /$ week of aerobic exercise), and mild to moderate impairment on the Short Physical Performance Battery (SPPB; scores 4-10). Potential participants had to agree to maintain their usual physical activity patterns and not to initiate any new exercise other than that associated with their randomly assigned treatment condition over the course of the study. Participants who were unwilling or unable to give informed consent, who were unwilling to accept random assignment, or who were participating in another research project were not accepted. Potential participants were excluded at screening if their medical history, clinical examination, or laboratory results revealed any of the following conditions: weight $>136.1 \mathrm{~kg}$, weight loss $>4.5 \mathrm{~kg}$ in the past 6 months, history of surgery for weight loss, hospitalization within the past 6 months, significant underlying disease likely to limit lifespan and/or increase risk of intervention (ie, coronary heart disease, chronic or recurrent respiratory or gastrointestinal conditions, cancer [except nonmelanoma skin cancer] within 5 years, fasting blood glucose $>110 \mathrm{mg} / \mathrm{dL}$, resting blood pressure $>160 / 90 \mathrm{mmHg}$ ) or bone, muscle, or joint conditions that would prevent walking on a regular basis. Potential participants were also excluded if they reported taking any of the following medications: antipsychotic agents, monoamine oxidase inhibitors, systemic corticosteroids, antibiotics for HIV or tuberculosis, chemotherapeutic drugs, or current use of prescription weight loss drugs.

\section{Ethics}

This study was approved by the University of Florida's Institutional Review Board (IRB Project \#399-2006). All participants provided written informed consent to participate in this study.

\section{Assessment procedures}

Participants were recruited between September 2006 and December 2008 through a variety of methods, including 
media articles, direct mailings, newspaper announcements, and presentations to community groups. Following telephone screening, potentially eligible people were invited to attend a screening visit during which the purposes and procedures of the study were explained and informed consent was obtained. After the participant provided consent, the following measurements were taken to determine eligibility: (i) physical examination including medical history and current medications; (ii) height, weight, and girth; (iii) electrocardiogram, resting heart rate, and blood pressure; and (iv) blood chemistries (metabolic profile, complete blood count, liver chemistries, and lipids). Data were collected at the University of Florida's Aging and Rehabilitation Research Center, and assessment staff members were blinded to participants' assigned conditions.

\section{Study design and procedure}

This was a single-blinded design where personnel responsible for testing were blinded to participants' randomized assignment. Eligible participants were randomized to the $\mathrm{WL}+\mathrm{E}$ intervention or to a waitlist educational control group. The $\mathrm{WL}+\mathrm{E}$ intervention targeted a $6 \%$ or greater weight loss through moderate changes in energy intake (ie, a reduction of 500-1000 kcal/day) coupled with exercise sessions during which participants engaged in both aerobic activities (ie, walking) and lower-body resistance training of moderate intensity. The educational control group consisted of a series of monthly lectures on health issues relevant to older adults but unrelated to weight loss, diet, or physical activity (eg, skin protection, sleep hygiene). The clinic examiners who measured outcomes were blinded to the treatment assignment. SAS's PROC PLAN (SAS Institute Inc., Cary, $\mathrm{NC}$, USA) was utilized for computerized randomization. ${ }^{16}$

\section{Interventions}

\section{Weight loss plus exercise $(W L+E)$ group}

In the WL+E group, participants attended a weekly groupbased weight management session and completed three structured exercise sessions each week. During the 60-minute weight management sessions, the group leaders provided participants with nutrition education and instruction in behavioral strategies (eg, self-monitoring, goal setting) designed to enhance adherence to dietary and exercise goals. Group problem solving was used when participants reported difficulty with meeting their weekly goals. Both the group and supervised exercise sessions were held in a community setting (ie, a church facility). Throughout the entire program, all intervention team members praised participants for success and used behavioral strategies to encourage adherence and improve retention.

\section{Dietary component}

Each participant's caloric assignment represented an approximate $750 \mathrm{kcal} /$ day deficit from her estimated energy intake at baseline, determined through analysis of food records. This $750 \mathrm{kcal} /$ day deficit was intended to promote weight loss at a rate of $0.7 \mathrm{~kg}$ per week. In line with the American Heart Association's dietary recommendations, ${ }^{17}$ the supervised weight loss program contained $55 \%, 30 \%$, and $15 \%$ of energy intake from carbohydrates, fats, and proteins, respectively. Food was self-selected under the supervision of a registered dietitian. Participants were instructed to complete daily food records, which they brought to each weight management group session. During these sessions, each participant's food record was reviewed by the registered dietitian and a doctoral student trained in behavioral science, who provided specific suggestions about dietary changes to help participants achieve their calorie goal.

\section{Exercise component}

The exercise intervention consisted of aerobic, strength training, and flexibility exercises. Walking was the primary mode of aerobic activity encouraged, but other forms of endurance activity (eg, stationary cycling) were also utilized when regular walking was contraindicated medically. After the third week of the intervention, participants were encouraged to meet a weekly walking goal of 150 minutes. Participants were supervised by exercise physiologists and certified personal trainers.

Throughout the intervention, participants attended three supervised exercise sessions each week. Blood pressure and heart rate were monitored before and after each supervised exercise session, which was preceded by a brief warm-up and followed by a cool-down period. Participants completed two 15-minute bouts of walking during each session. Following the first walking bout, participants were guided to complete a set of five lower-body exercises (ie, wide leg squat, standing leg curl, knee extension, side hip raise, and toe stand) during a 15-minute strength training routine. For each exercise, participants were encouraged to perform 10 repetitions (one set), rest for 1 minute, and then perform a second set. Adjustable ankle weights (up to $2.27 \mathrm{~kg}$ ) were used to provide increasing levels of resistance. For the leg curl, knee extension, and side hip raise exercise, the participants were instructed to perform a set of 10 repetitions with each leg before resting. Following their 
second walking bout, participants completed a 5-minute cool-down period during which they completed a series of flexibility exercises.

\section{Intensity of training}

The participants were introduced to the intervention exercises in a structured way such that they began with lighter-intensity exercise and gradually increased the intensity level over the first 2-3 weeks of the intervention. Following the initial adoption phase, participants were instructed to begin walking at a moderate intensity level. The Borg Perceived Exertion scale, ${ }^{18}$ a 15 -point self-assessment tool that ranges from 6 to 20, was used to help participants estimate the intensity level at which they were exercising. Participants were asked to walk at an intensity level of 13 (activity perception "somewhat hard"), and they were discouraged from exercising at levels that exceeded 15 ("hard") or dropped to 11 ("fairly light") or below. For the strength training component, participants were encouraged to complete each exercise at an intensity level that corresponded to a 15 or 16 ("hard").

\section{Educational control group}

The participants in the educational control group were asked to maintain their usual eating and physical activity patterns and not to engage in any intentional effort aimed at weight loss for 6 months. During the intervention, participants in this group attended monthly health education lectures on topics relevant to older adults that were not related to weight loss, diet, or physical activity (eg, skin protection, sleep hygiene). Following their 6-month assessments, participants in this group were offered the opportunity to receive the full 24-week WL+E intervention.

\section{Outcomes}

\section{Primary outcome: walking speed}

Walking speed (400-meter walk test)

Walking speed was assessed by the 400-meter walk test, during which participants were asked to complete a standard walking course at their usual pace. Participants were permitted to stop during the walk but were not allowed to sit or receive help from others and were required to complete the course in 15 minutes.

\section{Secondary outcomes: anthropometric and physical function measures \\ Body weight}

Body weight was taken in a fasting state and following voiding in the morning.

\section{SPPB}

The SPPB consists of a 4-meter walk, repeated chair stands, and three hierarchical standing balance tests. ${ }^{19}$ Walking speed was assessed by instructing participants to walk at their usual pace for a distance of 4 meters. For the chair test, participants were instructed to fold their arms across their chest and to stand up from a sitting position five times as quickly as possible. The time it took participants to complete this task was recorded. For the balance test, participants were instructed to maintain their feet in side by side, semitandem (heel of one foot beside the big toe of the other foot), and tandem (heel of one foot in front and touching the other foot) positions for 10 seconds each. The time to complete each of the three performance measures was assigned a categorical score based on normative data, ${ }^{7}$ ranging from 0 to 4 , with 4 indicating the highest level of performance and 0 the inability to complete the test. A summary score ranging from 0 (worst performers) to 12 (best performers) was calculated by adding walking speed, chair stands, and balance scores.

\section{Knee extension isokinetic strength}

Maximal knee extension strength using each participant's strongest leg was measured using an isokinetic dynamometer (Biodex, Shirley, NY). ${ }^{20}$ The participants were asked to develop their maximal isokinetic knee extension strength. Three trials of five repetitions were performed, and the peak torque value was used for statistical analyses.

\section{Statistical methods}

This trial represented a pilot study that was designed to demonstrate the feasibility, acceptability, and efficacy of the intervention; therefore, a power analysis was not conducted. The statistical analyses consisted of descriptive and intent-to-treat (ITT) modeling procedures. Post-treatment responses for dropouts were filled in using multiple imputation. The five imputed responses sampled from a normal distribution with the mean baseline value ("centered" with a baseline carried forward mechanism) and the conditional variance of the post-treatment value were given the pretreatment value. The means and standard deviations of variables were computed at baseline and at the end of the study; for responses with missing values, the means and standard errors (SE) were reported after multiple imputation. Change from baseline was defined as the value at time $t$ minus the value observed at baseline for all response measures. The main outcomes of interest were change from baseline to 6 months for (i) body weight, (ii) walking speed (assessed by the 400-meter walk test), (iii) the SPPB, and (iv) knee extension isokinetic strength. Differences in the change from baseline between the two treatment groups were tested 
using a multivariate regression with changes from baseline in body weight, walking speed, and muscle strength being the dependent variables in one model and the intervention group and the baseline value as the independent variables. Change in SPPB from baseline was compared between two groups with the Wilcoxon rank-sum test. Similar analyses were conducted to examine racial differences within the active treatment arm. Adjustments to variances were carried out using standard multiple imputation approaches. All analyses were conducted using the SAS Version 9.12 software package.

\section{Results}

Participant flow during the trial is outlined in Figure 1, and the descriptive characteristics of participants by condition are summarized in Table 1. One participant in the Educational
Control group dropped out of the study due to scheduling conflicts, and one participant in the WL+E group dropped out due to personal health issues unrelated to the intervention. The WL+E and educational control groups $(\mathrm{N}=34)$ were balanced in baseline characteristics. Three participants assigned to the WL+E intervention had diabetes, but no participants in the control group were diagnosed with diabetes. The participants were all nonsmokers. The majority of participants, 25 of 34, reported that their health was "good", six participants described their health as "fair", and three participants reported that they were in "excellent" health. The sample was evenly divided between African American $(n=18$ out of 34$)$ and Caucasian participants $(n=16$ out of 34$)$.

We used the ITT procedure in the analysis, such that participants' outcomes were compared based on their

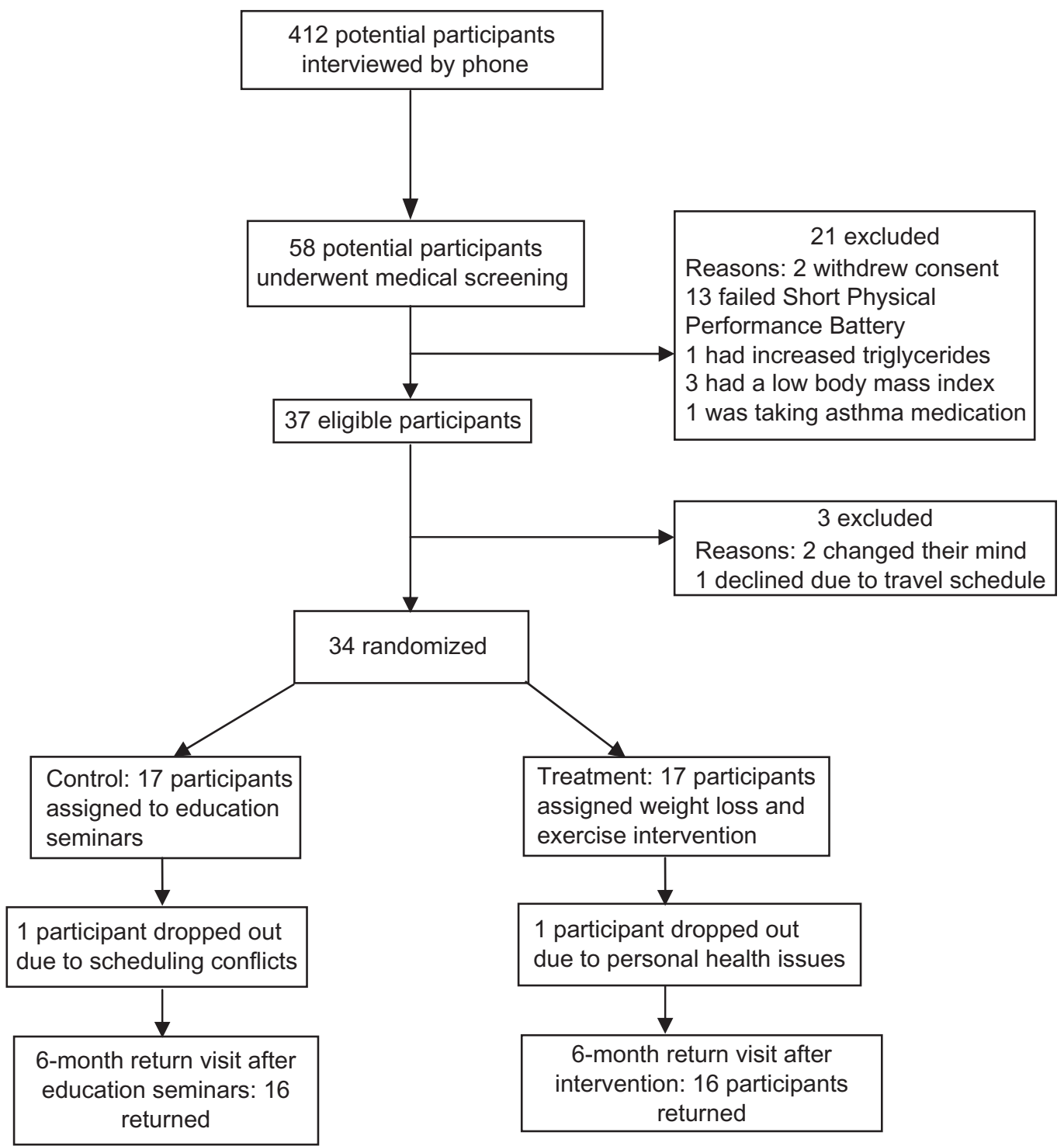

Figure I Participant flow during the trial. 
Table I Baseline characteristics of study participants

\begin{tabular}{|c|c|c|c|c|c|c|c|}
\hline & \multicolumn{3}{|c|}{ Educational control group } & \multicolumn{4}{|c|}{ Weight loss plus exercise group } \\
\hline & $\begin{array}{l}\text { Caucasian } \\
(n=8)\end{array}$ & $\begin{array}{l}\text { African American } \\
(n=9)\end{array}$ & $\begin{array}{l}\text { Total group } \\
(\mathrm{n}=17)\end{array}$ & $\begin{array}{l}\text { Caucasian } \\
(n=8)\end{array}$ & $\begin{array}{l}\text { African American } \\
(n=9)\end{array}$ & $\begin{array}{l}\text { Total group } \\
(\mathrm{n}=17)\end{array}$ & $P$ value $^{\mathrm{a}}$ \\
\hline & $\mathbf{M} \pm \mathbf{S D}$ & $\mathbf{M} \pm \mathbf{S D}$ & $\mathbf{M} \pm \mathbf{S D}$ & $\mathbf{M} \pm \mathbf{S D}$ & $\mathbf{M} \pm \mathbf{S D}$ & $\mathbf{M} \pm \mathbf{S D}$ & \\
\hline Age (years) & $67.1 \pm 8.2$ & $60.7 \pm 2.7$ & $63.7 \pm 6.7$ & $63.1 \pm 4.4$ & $64.2 \pm 4.8$ & $63.7 \pm 4.5$ & 1.0 \\
\hline Education (years) & $15.5 \pm 2.6$ & $13.3 \pm 3.3$ & $14.4 \pm 3.1$ & $14.8 \pm 2.4$ & $14.2 \pm 2.3$ & $14.5 \pm 2.3$ & 0.90 \\
\hline Weight $(\mathrm{kg})$ & $85.0 \pm 14.6$ & $95.6 \pm 17.6$ & $90.6 \pm 16.6$ & $93.1 \pm 11.9$ & $104.7 \pm 26.3$ & $99.3 \pm 21.1$ & 0.19 \\
\hline Body mass index $\left(\mathrm{kg} / \mathrm{m}^{2}\right)$ & $33.1 \pm 3.1$ & $38.3 \pm 8.3$ & $35.8 \pm 6.8$ & $36.5 \pm 2.4$ & $39.1 \pm 7.3$ & $37.8 \pm 5.5$ & 0.36 \\
\hline SPPB score & $9.0 \pm 1.1$ & $9.1 \pm 1.1$ & $9.1 \pm 1.1$ & $9.3 \pm 1.0$ & $9.1 \pm 0.8$ & $9.2 \pm 0.8$ & 0.71 \\
\hline 400-meter walk & $0.95 \pm 0.4$ & $0.97 \pm 0.17$ & $0.96 \pm 0.3$ & $0.91 \pm 0.15$ & $0.91 \pm 0.36$ & $0.91 \pm 0.27$ & 0.61 \\
\hline Leg extension strength, lb & $73.4 \pm 14.5$ & $82.1 \pm 19.5$ & $78.0 \pm 17.4$ & $68.7 \pm 14.3$ & $68.0 \pm 23.3$ & $68.3 \pm 19.0$ & 0.13 \\
\hline \multicolumn{8}{|l|}{ Self-reported health, $n$ (\%) } \\
\hline Excellent & I (I3\%) & $0(0 \%)$ & I (6\%) & $0(0 \%)$ & $2(22 \%)$ & $2(12 \%)$ & \\
\hline Good & $5(62 \%)$ & 7 (78\%) & $12(70 \%)$ & 7 (87\%) & $6(67 \%)$ & $13(76 \%)$ & \\
\hline Fair & $2(25 \%)$ & $2(22 \%)$ & $4(24 \%)$ & I (I3\%) & I (II\%) & $2(12 \%)$ & \\
\hline
\end{tabular}

Note: a $P$ value based on total group comparison: weight loss plus exercise group versus educational control group.

Abbreviations: M, mean; SD, standard deviation; SPPB, short physical performance battery.

randomized groups regardless of their compliance to the intervention. The two groups were significantly different in outcome changes overall (multivariate regression $\chi^{2}$ $(3=27.7 ; P<0.001)$. Changes in each of these outcome variables are described below.

\section{Outcomes and estimation}

\section{Primary outcome: walking speed}

Walking speed (400-meter walk test)

The walking speed (meters/second $[\mathrm{m} / \mathrm{s}]$ ) of participants in the WL+E group significantly increased compared to the control group $($ mean $[\mathrm{SE}]=0.16[0.03] \mathrm{m} / \mathrm{s}$ vs $0.02[0.03]$ $\mathrm{m} / \mathrm{s} ; P=0.016 ;$ mean difference $=0.14 ; 95 \%$ confidence interval $[\mathrm{CI}] 0.04,0.24)$. Within the WL+E group, the African American participants had a mean increase in walking speed of $0.13(0.05) \mathrm{m} / \mathrm{s}$ and Caucasian participants had an improvement of $0.19(0.05) \mathrm{m} / \mathrm{s}$. This difference was not statistically significant $(P>0.05)$.

\section{Secondary outcomes: anthropometric and physical function measures \\ Body weight}

Participants randomized to the WL+E group lost significantly more weight than did participants in the control group (mean $[\mathrm{SE}]=5.95[0.99] \mathrm{kg}$ vs $0.23[0.99] \mathrm{kg} ; P=0.004$; mean difference $=5.72 ; 95 \%$ CI 2.82, 8.6). The African American participants lost $6.50(1.39) \mathrm{kg}, 5.6 \%$ of baseline weight, whereas the Caucasian participants lost $5.36(1.45) \mathrm{kg}, 5.9 \%$ of baseline weight, during the intervention; this difference was not statistically significant $(P=0.48)$.

\section{Physical function \\ SPPB}

Scores on the SPPB improved in both the WL+E and control groups (mean change $[\mathrm{SE}]$ in $\mathrm{WL}+\mathrm{E}$ group $=1.82$ [0.36]; $P<0.001 ;$ mean change in the control group $=0.8$ [0.29]; $P<0.05$; mean difference $=1.02 ; 95 \%$ CI: 0.16 , $1.88 ; P=0.02$ ). Within the WL+E group, the African American participants and Caucasian participants had similar improvements in scores on the SPPB (mean change [SE] = 1.61 [0.37] versus 1.94 [0.42]).

\section{Knee extension strength}

Knee extension strength did not significantly change from baseline in either the WL+E or control group, and there were no significant differences between groups (mean improvement in strength [SE] for WL+E group $=4.33$ [3.98] vs 3.95 [3.5] $\mathrm{kg}$ for control group; mean difference $=0.38 ; 95 \%$ CI 10.5 , 10.91). Within the WL+E group, there was an improvement in knee extension strength in the African American participants but not in the Caucasian participants (mean improvement in strength [SE] for African American participants = 12.36 [4.76] vs -4.13 [4.89] kg for Caucasian participants), but this difference was not statistically significant. Table 2 displays changes in all outcome variables for this study.

\section{Adherence}

Mean attendance (standard deviation [SD]) at group weight loss sessions was $83 \%(16 \%)$, and mean attendance at exercise sessions was $70 \%$ (26\%). Participants met the walking goal of the program and reported walking an average of 161 minutes 
Table 2 Main outcome results: adjusted and unadjusted change between baseline and 6-month assessment

\begin{tabular}{|c|c|c|c|c|c|c|c|}
\hline & \multicolumn{3}{|c|}{ Educational control } & \multicolumn{4}{|c|}{ Weight loss plus exercise group } \\
\hline & $\begin{array}{l}\text { Caucasian } \\
(n=8)\end{array}$ & $\begin{array}{l}\text { African American } \\
(\mathrm{n}=9)\end{array}$ & $\begin{array}{l}\text { Total group } \\
(n=17)\end{array}$ & $\begin{array}{l}\text { Caucasian } \\
(\mathrm{n}=8)\end{array}$ & $\begin{array}{l}\text { African American } \\
(\mathrm{n}=9)\end{array}$ & $\begin{array}{l}\text { Total group } \\
(n=17)\end{array}$ & $P$ value \\
\hline & $M \pm S D$ & $M \pm S D$ & $\mathbf{M} \pm \mathbf{S D}$ & $\mathbf{M} \pm \mathbf{S D}$ & $M \pm S D$ & $\mathbf{M} \pm \mathbf{S D}$ & \\
\hline \multicolumn{8}{|l|}{ Unadjusted outcomes } \\
\hline Weight (kg) & $-0.89 \pm 4.12$ & $0.09 \pm 3.91$ & $-0.37 \pm 3.92$ & $-5.45 \pm 3.24$ & $-6.18 \pm 4.42$ & $-5.84 \pm 3.81$ & 0.001 \\
\hline SPPB score & $1.88 \pm 1.46$ & $0.22 \pm 1.86$ & $1.00 \pm 1.84$ & $1.63 \pm 0.92$ & $1.22 \pm 1.72$ & $1.41 \pm 1.37$ & 0.46 \\
\hline 400-meter walk & $0.03 \pm 0.14$ & $0.04 \pm 0.11$ & $0.03 \pm 0.12$ & $0.19 \pm 0.21$ & $0.11 \pm 0.13$ & $0.15 \pm 0.17$ & 0.03 \\
\hline Leg strength (kg) & $-0.74 \pm 6.74$ & $4.52 \pm 10.59$ & $2.74 \pm 8.93$ & $-1.13 \pm 12.74$ & $12.68 \pm 25.40$ & $6.18 \pm 21.07$ & 0.54 \\
\hline \multicolumn{8}{|l|}{ Adjusted outcomes } \\
\hline Weight (kg) & $-0.47 \pm 4.44$ & $-0.05 \pm 4.35$ & $-0.23 \pm 4.08$ & $-5.36 \pm 4.10$ & $-6.50 \pm 4.17$ & $-5.95 \pm 4.08$ & 0.004 \\
\hline SPPB score & $1.81 \pm 1.88$ & $-0.17 \pm 1.17$ & $0.80 \pm 1.20$ & $1.94 \pm 1.19$ & $1.61 \pm 1.11$ & $1.82 \pm 1.24$ & 0.02 \\
\hline 400-meter walk & $0.01 \pm 0.14$ & $0.03 \pm 0.15$ & $0.02 \pm 0.12$ & $0.19 \pm 0.14$ & $0.13 \pm 0.15$ & $0.16 \pm 0.12$ & 0.009 \\
\hline Leg strength $(\mathrm{kg})$ & $2.14 \pm 15.30$ & $6.27 \pm 14.58$ & $3.95 \pm 14.43$ & $-4.13 \pm 13.83$ & $12.36 \pm 14.28$ & $4.33 \pm 14.35$ & 0.94 \\
\hline
\end{tabular}

Notes: ' $P$ value based on total group comparison; $W L+E$ vs. educational control group. Adjusted change: control for age, race, bmi, education, group, and group*race. Abbreviations: M, mean; SD, standard deviation; SPPB, short physical performance battery.

per week. The African American participants attended 17.5 of the 23 group weight loss sessions, whereas the Caucasian participants attended 20 of the 23 group weight loss sessions. The African American participants attended fewer exercise sessions than did the Caucasian participants (42 vs 59 sessions) but reported walking more minutes per week than the Caucasian participants (192 vs 126 weekly minutes). Both the African American and Caucasian participants met their calorie goals $50 \%$ of the time and completed food records on average 5 out of 7 days per week (mean [SD] for African American participants $=5.0[1.5] ;$ Caucasian participants $=5.1[0.9]$ )

\section{Adverse events}

Less than half of the participants $(n=12$ out of 34$)$ reported experiencing bone, muscle, or joint pain during the study. There was not a significant difference in the number of participants who reported experiencing musculoskeletal problems in the WL+E $(\mathrm{n}=7)$ and Educational Control groups $(n=5)$. No participants experienced any adverse changes in blood chemistry, renal, or liver function test results.

\section{Discussion}

The major finding of this study was that a lifestyle-based weight loss plus exercise intervention produced significant weight loss and improved physical function in obese, older women with mild to moderate physical impairments. Participants in the intervention group lost approximately $6 \%$ of their initial body weight and significantly improved physical function as measured by changes in gait, balance, transfers, and walking speed. In line with previous studies, ${ }^{21}$ participants in the educational control group also significantly improved their physical function, as measured by performance on the SPPB; the observed improvements on the SPPB in the Educational Control group may have been related to practice effects. Therefore, the difference in the improvement in scores between the two groups may represent the additive benefit of a lifestyle-based weight loss intervention. Muscle strength was also maintained in both the intervention and control groups.

Older adults may be particularly susceptible to the adverse effects of excessive body weight on physical function because of the decrease in muscle mass and strength that occurs with aging. ${ }^{22} \mathrm{~A}$ significant concern about encouraging weight loss in this population is that it could have a detrimental impact on muscle function and strength. We did not observe adverse effects on muscle function in the present study, as muscle strength was maintained among participants in the intervention group. Because participants in the intervention group maintained strength despite losing a significant amount of weight, this suggests that muscle quality (ie, strength/muscle volume) may have improved. Therefore, the findings of the present study are encouraging regarding the potential of lifestyle interventions to delay or prevent the development of disability in obese, older women. These findings are similar to one previous clinical trial that found that a lifestyle-based diet plus exercise intervention was effective in improving physical function in frail, obese, older adults. ${ }^{12}$

Given the disproportionately higher rates of obesity $(>50 \%)$ and obesity-related comorbidities in African American women, ${ }^{1,2}$ interventions are urgently needed 
that can effectively reduce body weight and improve physical function levels in this high-risk population. To our knowledge, this is the first study to examine the effects of a lifestyle-based weight loss plus exercise intervention in obese, older African American women. In contrast to previous studies in younger and middle-aged African American women, ${ }^{23,24}$ we found that obese, older, African American women responded in a similar manner as obese, older, Caucasian women in terms of weight loss and improvements in physical function. The specific reason(s) for the divergence in findings from previous studies is unclear, but a number of factors may have influenced the effectiveness of our intervention in African American women, including the intervention setting (ie, church facilities), inclusion of a structured group-based exercise program, and a tailored dietary intervention approach, as well as participant characteristics (ie, age, motivation, health risk). Interestingly, among African American women who do achieve and sustain weight loss, significant improvements in cardiovascular disease risk factors have been observed, ${ }^{25}$ suggesting that weight loss may be effective in reducing the risk of chronic disease in this high-risk population.

The results of the present study should be interpreted in the context of its limitations. First, the sample size was relatively small, and the study was not adequately powered to detect differences between African American and Caucasian women in response to the intervention. Second, we did not directly measure body composition and therefore were unable to determine the proportion of fat versus fatfree weight lost. Third, participants completed just over two-thirds of the center-based exercise sessions (mean attendance $=70 \%$ ). Although this exercise completion was lower than was anticipated, it is in line with previous studies that have found that participants complete approximately two-thirds of exercise prescribed, regardless of frequency recommended. ${ }^{11,26}$ The reasons for the lower than anticipated rates of attendance at exercise sessions were varied. Some participants reported being unable to attend exercise sessions due to changes in their schedule, and others reported preferring to exercise at home. Fourth, this study informs only about the effects of a short-term (ie, 6-month) weight loss intervention, which is not of sufficient duration to determine whether weight loss is sustained. An additional limitation of this study is that the participants were generally healthy, older adults; therefore, these findings may not be generalizable to older adults with more severe health conditions or functional limitations.
The present study also had a number of strengths. Few studies have tested the effects of a comprehensive lifestyle-based weight loss intervention in an older adult population with impaired physical functioning. Given that participants had a mean age of 68 years, this study provides preliminary information regarding how adults over the age of 65 years may respond to a comprehensive weight loss plus exercise program. In addition, the patient-centered outcomes (ie, walking speed, strength, and SPPB) used in this study have high relevance to older adults and have been found to predict disability, falls, institutionalization, and mortality. Another strength of this study is the inclusion of equal numbers of African American and Caucasian obese women. Additionally, all exercise sessions were conducted under direct supervision to ensure that participants exercised at the appropriate intensity and used proper exercise techniques. Finally, the intervention was carried out in a community setting and thus represents a unique approach to implementing lifestyle-based interventions within communities.

\section{Conclusion}

The findings of this study suggest that a lifestyle-based weight loss program consisting of moderate caloric restriction plus moderate aerobic and resistance exercise can produce significant weight loss and improve indices of physical function while maintaining muscle strength in obese, older African American and Caucasian women with mild to moderate physical limitations. Future research with larger study samples over longer time periods is needed to further evaluate the effects of a lifestyle-based weight loss program in obese, older women from different racial backgrounds. Additionally, longer-term trials are needed to determine whether the observed gains are maintained with or without continued intervention.

\section{Acknowledgment}

The authors would like to express their appreciation to the participants and research associates who made it possible to complete this research project. This research was supported by the National Institutes of Health-Funded Claude D Pepper Older Americans Independence Center (P30AG028740). Stephen Anton is supported by a K23 AT004251-01A2, an Early Career Investigator Award from the American Heart Association (09CRP2390173), and the Thomas H Maren Foundation.

\section{Disclosure}

The authors report no conflicts of interest in this work. 


\section{References}

1. Hedley AA, Ogden CL, Johnson CL, et al. Prevalence of overweight and obesity among US children, adolescents, and adults, 1999-2002. JAMA. 2004;291:2847-2850.

2. Flegal KM, Carroll MD, Ogden CL, Curtin LR. Prevalence and trends in obesity among US adults, 1999-2008. JAMA. 2010;303:235-241.

3. Manson JE, Bassuk SS. Obesity in the United States: a fresh look at its high toll. JAMA. 2003;289:229-230.

4. Jones GL, Sutton A. Quality of life in obese postmenopausal women. Menopause Int. 2008;14:26-32.

5. Baumgartner RN. Body composition in healthy aging. Ann NY Acad Sci. 2000;904:437-448.

6. Blaum CS, Xue QL, Michelon E, et al. The association between obesity and the frailty syndrome in older women: the Women's Health and Aging Studies. J Am Geriatr Soc. 2005;53:927-934.

7. Forbes GB. Longitudinal changes in adult fat-free mass: influence of body weight. Am J Clin Nutr. 1999;70:1025-1031.

8. Houston DK, Nicklas BJ, Zizza CA. Weighty concerns: the growing prevalence of obesity among older adults. J Am Diet Assoc. 2009;109: 1886-1895.

9. Miller SL, Wolfe RR. The danger of weight loss in the elderly. J Nutr Health Aging. 2008;12:487-491.

10. Cesari M, Kritchevsky SB, Baumgartner RN, et al. Sarcopenia, obesity, and inflammation: results from the Trial of Angiotensin Converting Enzyme Inhibition and Novel Cardiovascular Risk Factors study. Am J Clin Nutr. 2005;82:428-434.

11. Witham MD, Avenell A. Interventions to achieve long-term weight loss in obese older people: a systematic review and meta-analysis. Age Ageing. 2010;39:176-184.

12. Villareal DT, Banks M, Sinacore DR, et al. Effect of weight loss and exercise on frailty in obese older adults. Arch Intern Med. 2006;166: 860-866

13. Davidson LE, Hudson R, Kilpatrick K, et al. Effects of exercise modality on insulin resistance and functional limitation in older adults: a randomized controlled trial. Arch Intern Med. 2009;169:122-131.

14. Chomentowski P, Dube JJ, Amati F, et al. Moderate exercise attenuates the loss of skeletal muscle mass that occurs with intentional caloric restriction-induced weight loss in older, overweight to obese adults. J Gerontol A Biol Sci Med Sci. 2009;64:575-580.
15. Frimel TN, Sinacore DR, Villareal DT. Exercise attenuates the weight-loss-induced reduction in muscle mass in frail obese older adults. Med Sci Sports Exerc. 2008;40:1213-1219.

16. Spector P, Goodnight J, Sall J, et al. The GLM procedure. SAS User's Guide:Statistics Version 5. Cary, NC: SAS Institute Inc.; 1985: 433-507.

17. Krauss RM, Eckel RH, Howard B, et al. AHA dietary guidelines: revision 2000: a statement for healthcare professionals from the Nutrition Committee of the American Heart Association. Circulation. 2000;102:2284-2299.

18. Borg GAV. Physical Performance and Perceived Exertion. Lund, Sweden: Gleerup; 1962.

19. Guralnik JM, Ferrucci L, Simonsick EM, et al. Lower-extremity function in persons over the age of 70 years as a predictor of subsequent disability. N Engl J Med. 1995;332:556-561.

20. Taylor NA, Sanders RH, Howick EI, Stanley SN. Static and dynamic assessment of the Biodex dynamometer. Eur J Appl Physiol Occup Physiol. 1991;62:180-188.

21. Espeland MA, Gill TM, Guralnik J, et al. Designing clinical trials of interventions for mobility disability: results from the Lifestyle Interventions and Independence for Elders Pilot (LIFE-P) trial. J Gerontol A Biol Sci Med Sci. 2007;62:1237-1243.

22. Roubenoff R. Sarcopenic obesity: the confluence of two epidemics. Obes Res. 2004;12:887-888.

23. Darga LL, Holden JH, Olson SM, Lucas CP. Comparison of cardiovascular risk factors in obese blacks and whites. Obes Res. 1994;2: 239-245.

24. West DS, Elaine PT, Bursac Z, Felix HC. Weight loss of black, white, and Hispanic men and women in the Diabetes Prevention Program Obesity (Silver Spring). 2008;16:1413-1420.

25. Walcott-McQuigg JA, Chen SP, Davis K, et al. Weight loss and weight loss maintenance in African American women. J Natl Med Assoc. 2002; 94:686-694.

26. Perri MG, Anton SD, Durning PE, et al. Adherence to exercise prescriptions: effects of prescribing moderate versus higher levels of intensity and frequency. Health Psychol. 2002;21:452-458.
Clinical Interventions in Aging

\section{Publish your work in this journal}

Clinical Interventions in Aging is an international, peer-reviewed journal focusing on evidence-based reports on the value or lack thereof of treatments intended to prevent or delay the onset of maladaptive correlates of aging in human beings. This journal is indexed on PubMed Central, MedLine, the American Chemical Society's 'Chemical Abstracts

\section{Dovepress}

Service' (CAS), Scopus and the Elsevier Bibliographic databases. The manuscript management system is completely online and includes a very quick and fair peer-review system, which is all easy to use. Visit $\mathrm{http}: / / \mathrm{ww}$.dovepress.com/testimonials.php to read real quotes from published authors. 